\title{
Strangeness production in high multiplicity pp collisions
}

\section{Marek Bombara* for the ALICE Collaboration}

Pavol Jozef Safarik University

E-mail: marek.bombara@cern.ch

\begin{abstract}
Over the last three decades, proton-proton ( $\mathrm{pp}$ ) collisions have successfully provided a reference sample for the study of nucleus-nucleus collisions and have helped to unveil features attributed there to the formation of Quark Gluon Plasma. The results from the LHC pp program revealed some intriguing features in pp collisions that resemble those of nucleus-nucleus collisions and therefore bring into question the reference sample paradigm at the $\mathrm{TeV}$ scale. One of the measured effects is strangeness enhancement as function of multiplicity, where similar production rates of (multi-)strange particles are observed in high multiplicity pp collisions as in peripheral $\mathrm{Pb}-\mathrm{Pb}$ collisions. A review of the multiplicity dependence of strange and multi-strange particle production in pp collisions at 7 and $13 \mathrm{TeV}$ measured by the ALICE experiment is reported.
\end{abstract}

Critical Point and Onset of Deconfinement

7-11 August, 2017

The Wang Center, Stony Brook University, Stony Brook, NY

${ }^{*}$ Speaker. 


\section{Introduction}

An enhanced production of strangeness relative to $\mathrm{pp}$ collisions was proposed among the first signatures of deconfined quark matter in nucleus-nucleus collisions [1]. In order to quantify the signature experimentally, the enhancement was measured as the yield of strange particles normalized to the mean number of participating nucleons $\left(\left\langle N_{\text {part }}\right\rangle\right)$ with respect to the same quantity in a reference sample (pp or $\mathrm{p}-\mathrm{A}$ collisions). Defined in this way, strangeness enhancement was indeed observed for different collision energies at SPS [2], RHIC [3] and LHC [4], increasing with the system size and with the strangeness content of the hyperon. In a more modern view, the measured effect has been explained as a supression of strangeness production in the reference sample due to the lack of phase space for strange quark creation in small systems [5].

First multiplicity dependent studies at LHC in small colliding systems (pp and p-Pb) unveiled striking features usually attributed to medium collectivity in nucleus-nucleus collisions $[6,7,8]$. In this paper, previously unexpected trends in strangeness production in high multiplicity pp collisions measured by the ALICE experiment are reported [9].

\section{Analysis and results}

The results presented here are based on the analysis of $10^{8} \mathrm{pp}$ collisions collected in 2010 $(\sqrt{s}=7 \mathrm{TeV})$ and $5 \times 10^{7} \mathrm{pp}$ collisions collected in $2015(\sqrt{s}=13 \mathrm{TeV})$. A detailed description of the ALICE detector can be found in [10]. Analysed events were collected using a minimumbias trigger and are selected to have at least one charged particle in $|\eta|<1$ (INEL>0). The event sample was divided into 10 multiplicity classes according to ionisation energy (proportional to the charged particle multiplicity) deposited in VZERO detectors, covering the regions $2.8<\eta<5.1$ and $-3.7<\eta<-1.7$. The corresponding average pseudorapidity density of primary charged particles $\left(\left\langle d N_{\mathrm{ch}} / d \eta\right\rangle\right)$ measured at mid-rapidity and the fractions of total visible hadronic cross section in ALICE are listed in Table 1.

The (multi-)strange particle candidates $\mathrm{K}_{S}^{0}, \Lambda(\bar{\Lambda}), \Xi^{-}\left(\bar{\Xi}^{+}\right)$and $\Omega^{-}\left(\bar{\Omega}^{+}\right)$are identified via their

Table 1: Event multiplicity classes, their corresponding fraction of the INEL $>0$ cross-section $\left(\sigma / \sigma_{\mathrm{INEL}}>0\right)$ and their corresponding $\left\langle d N_{\mathrm{ch}} / d \eta\right\rangle$ at midrapidity $(|\eta|<0.5)$ [9].

\begin{tabular}{cccccc}
\hline Class name & I & II & III & IV & V \\
\hline$\sigma / \sigma_{\text {INEL }}>0$ & $0-0.95 \%$ & $0.95-4.7 \%$ & $4.7-9.5 \%$ & $9.5-14 \%$ & $14-19 \%$ \\
$\left\langle d N_{\text {ch }} / d \eta\right\rangle$ & $21.3 \pm 0.6$ & $16.5 \pm 0.5$ & $13.5 \pm 0.4$ & $11.5 \pm 0.3$ & $10.1 \pm 0.3$ \\
\hline Class name & VI & VII & VIII & IX & X \\
\hline$\sigma / \sigma_{\text {INEL }}>0$ & $19-28 \%$ & $28-38 \%$ & $38-48 \%$ & $48-68 \%$ & $68-100 \%$ \\
$\left\langle d N_{\text {ch }} / d \eta\right\rangle$ & $8.45 \pm 0.25$ & $6.72 \pm 0.21$ & $5.40 \pm 0.17$ & $3.90 \pm 0.14$ & $2.26 \pm 0.12$ \\
\hline
\end{tabular}

characteristic decay topology using tracking detectors, namely the silicon Inner Tracking System (ITS) and Time Projection Chamber (TPC). Geometrical selection criteria were optimized and applied on the candidates in order to distinguish between signal and background. The particle yield was extracted by analysing invariant mass distribution of the decay products.

The $p_{\mathrm{T}}$ spectra of strange and non-strange light flavour hadrons measured at mid-rapidity $(|y|<0.5)$ are shown in Figure 1. The spectra are observed to become harder as the multiplicity 
increases. In nucleus-nucleus collisions this feature is usually understood as due to the presence of radial flow (collective expansion of the strongly interacting medium).

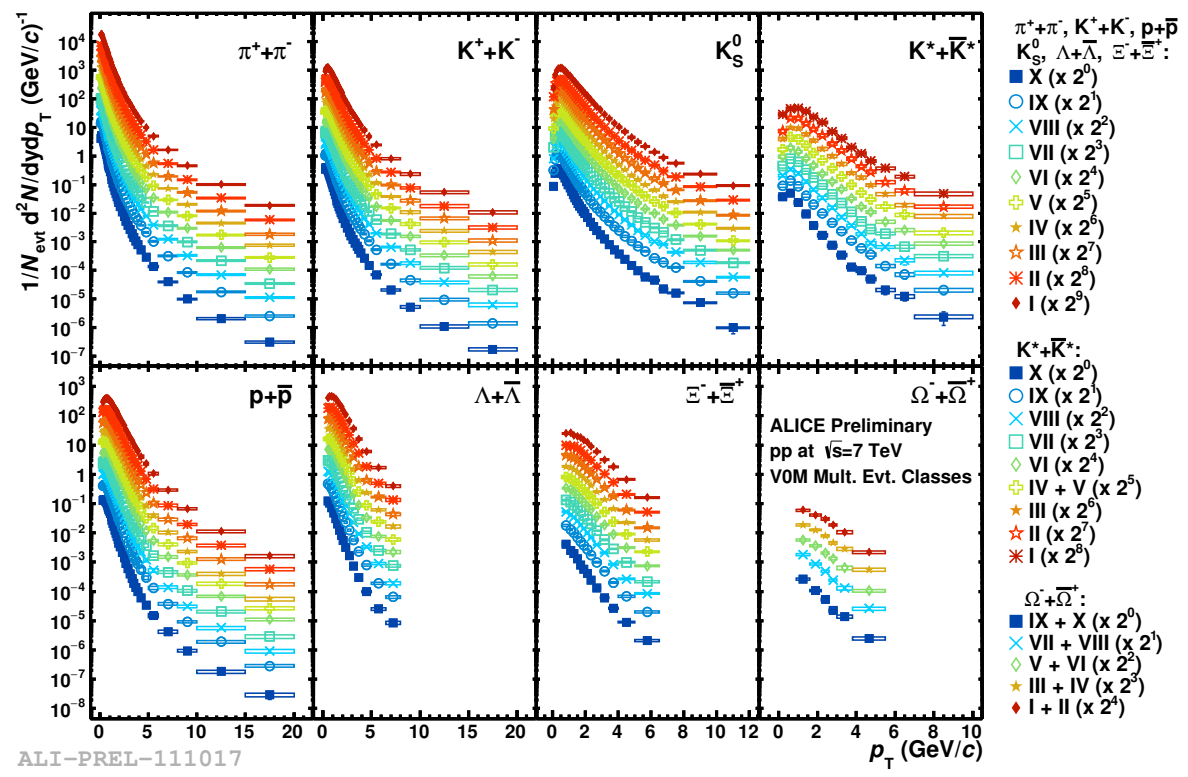

Figure 1: $p_{\mathrm{T}}$ spectra of identified hadrons for various multiplicity classes in pp collisions at $\sqrt{s}=7 \mathrm{TeV}$.

Another interesting feature was observed in $\mathrm{Pb}-\mathrm{Pb}$ collisions by studying baryon-to-meson ratios as a function of $p_{\mathrm{T}}$ : the maximum of the ratio is higher in central events with respect to peripheral events and it is shifted towards higher $p_{\text {T }}$ pointing to the increase of radial flow from peripheral to central collisions (Fig. 2, right) [11]. A qualitatively similar behaviour is observed also for $\mathrm{pp}$ and $\mathrm{p}-\mathrm{Pb}$ collisions while comparing the baryon-to-meson ratio in high and low multiplicity collisions (Fig. 2, left and centre).

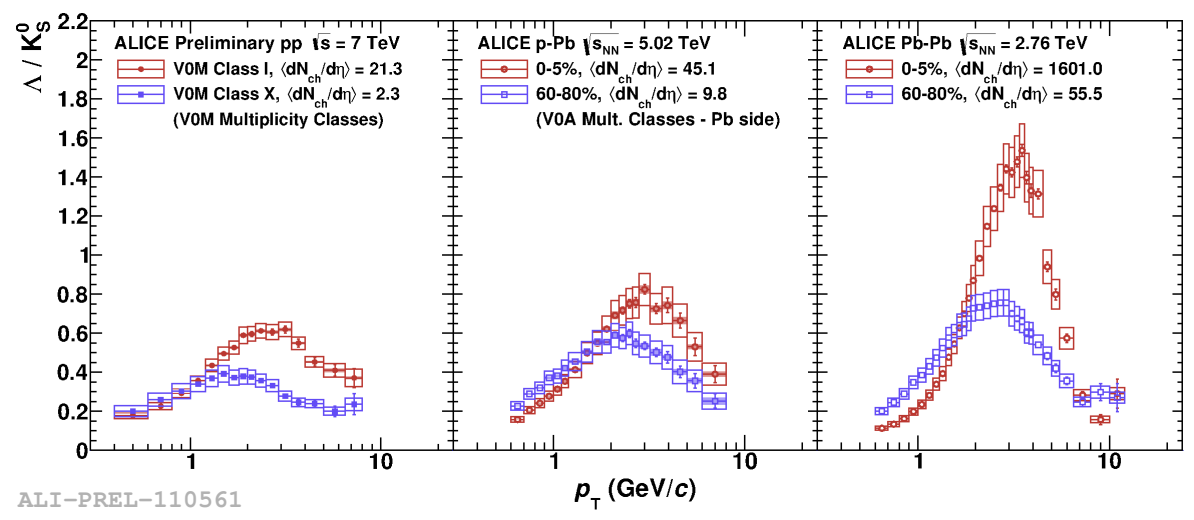

Figure 2: $p_{\mathrm{T}}$-differential $\Lambda / K_{S}^{0}$ ratio as a function of $p_{\mathrm{T}}$ for lowest and highest multiplicity classes in pp (left), $\mathrm{p}-\mathrm{Pb}$ (centre) and $\mathrm{Pb}-\mathrm{Pb}$ (right) collisions.

Since charged particle production does not scale linearly with $\left\langle N_{\text {part }}\right\rangle$ at the LHC [12], a better observable to quantify strangeness enhancement is the ratio of strange particle production to non- 
strange particle production (e.g. pions) for the same multiplicity or $\left\langle N_{\text {part }}\right\rangle$. In Figure 3, the ratios to pions are plotted as a function of multiplicity. The high multiplicity $\mathrm{pp}$ and $\mathrm{p}-\mathrm{Pb}$ ratios reach values of the same magnitude as in $\mathrm{Pb}-\mathrm{Pb}$ and the ratios suggest a saturation trend for central $\mathrm{Pb}-$ $\mathrm{Pb}$ events. The enhancement is clearly visible with increasing multiplicity in pp collisions. Also, the particle ratios in $\mathrm{p}-\mathrm{Pb}$ are found to be identical within uncertainties to those in peripheral $\mathrm{Pb}-\mathrm{Pb}$ collisions at similar multiplicities which points to the production being independent on colliding system at a given multiplicity.

Looking at the same observable at the RHIC and LHC energies, we observe a remarkable overlap

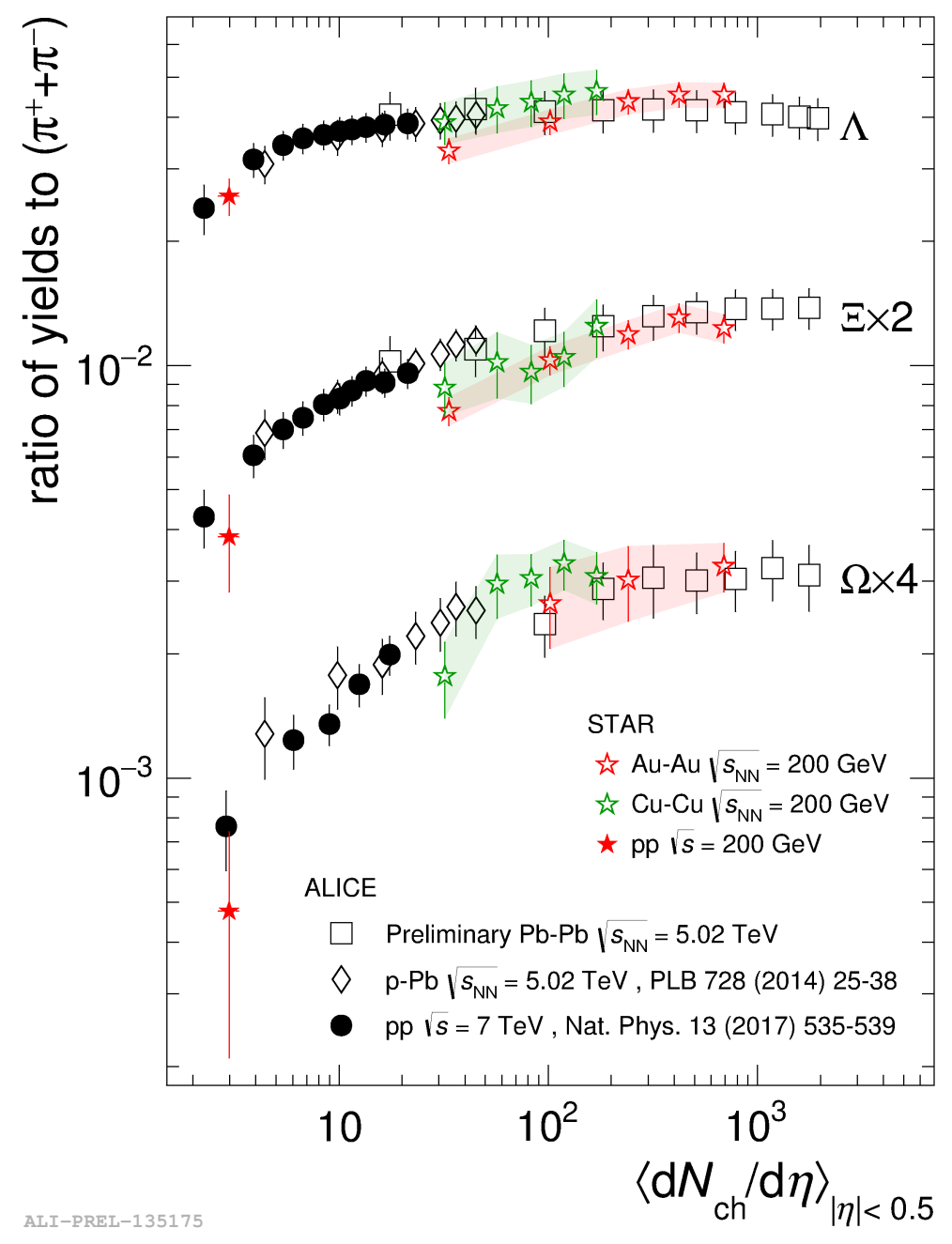

Figure 3: $p_{\mathrm{T}}$-integrated yield ratio to pions as a function of $\left\langle d N_{\mathrm{ch}} / d \eta\right\rangle$ measured in $|y|<0.5$

of $\mathrm{p}-\mathrm{Pb}$ and peripheral $\mathrm{Pb}-\mathrm{Pb}$ at $\mathrm{LHC}$ with $\mathrm{Cu}-\mathrm{Cu}$ and $\mathrm{Au}-\mathrm{Au}$ at $\mathrm{RHIC}[14,15]$, i.e. even for 25 times smaller collision energy the strangeness production near mid-rapidity is similar. The multiplicity or system size seems to drive the chemical composition of the system, not the collision energy.

Strange particle-to-pion ratios normalized to the ratio in the multiplicity-inclusive sample as a function of multiplicity in pp and $\mathrm{p}-\mathrm{Pb}$ are shown in Fig. 4 [9]. We observe the strangeness 


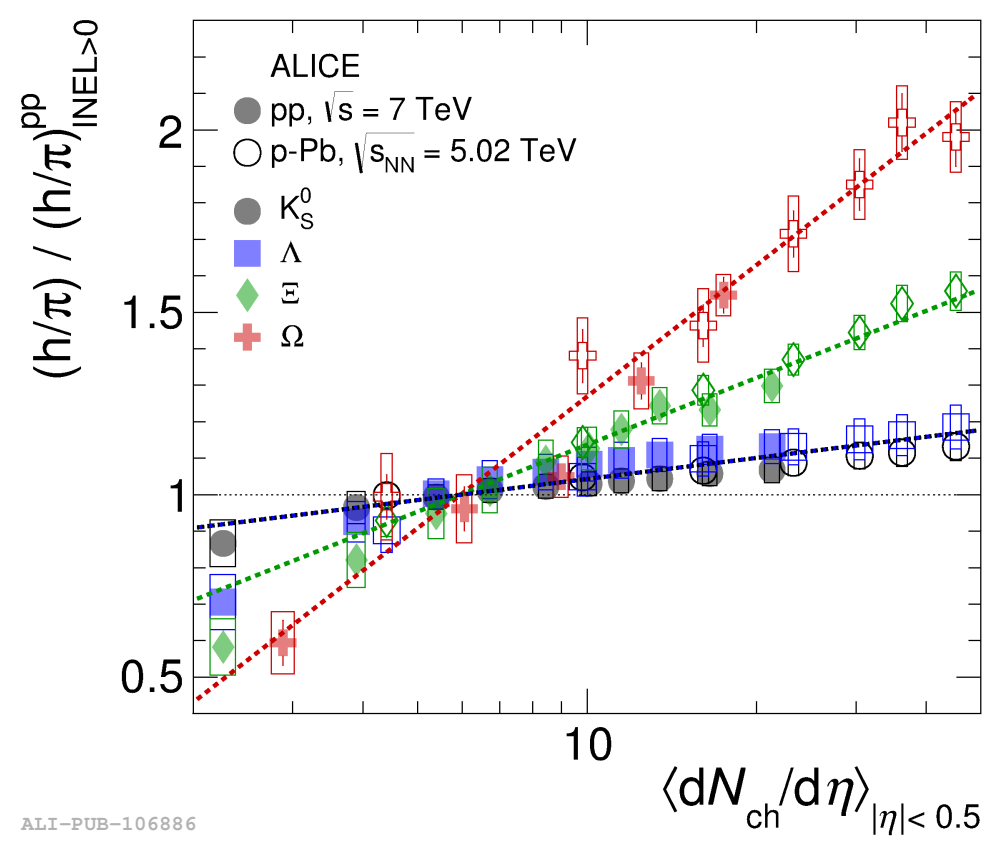

Figure 4: Particle yield ratios to pions of strange and multi-strange hadrons normalised to the values measured in the inclusive $I N E L>0 \mathrm{pp}$ sample, both in $\mathrm{pp}$ and in $\mathrm{p}-\mathrm{Pb}$ collisions.

enhancement rising with the strangeness content of the hadron. The results for $\Lambda$ and $\mathrm{K}_{S}^{0}$ overlap confirming the enhancement pattern is not caused by the mass differences among the strange particles.

For the strangeness production in pp at $\sqrt{s}=13 \mathrm{TeV}$ similar trends can be observed namely a hardening of the spectra with increasing multiplicity, evident in the increase of the mean $p_{\mathrm{T}}$ (Fig. 5, left). We also do not observe a dependence on the collision energy for integrated yields as a function of multiplicity for all identified strange particles (Fig. 5, right).
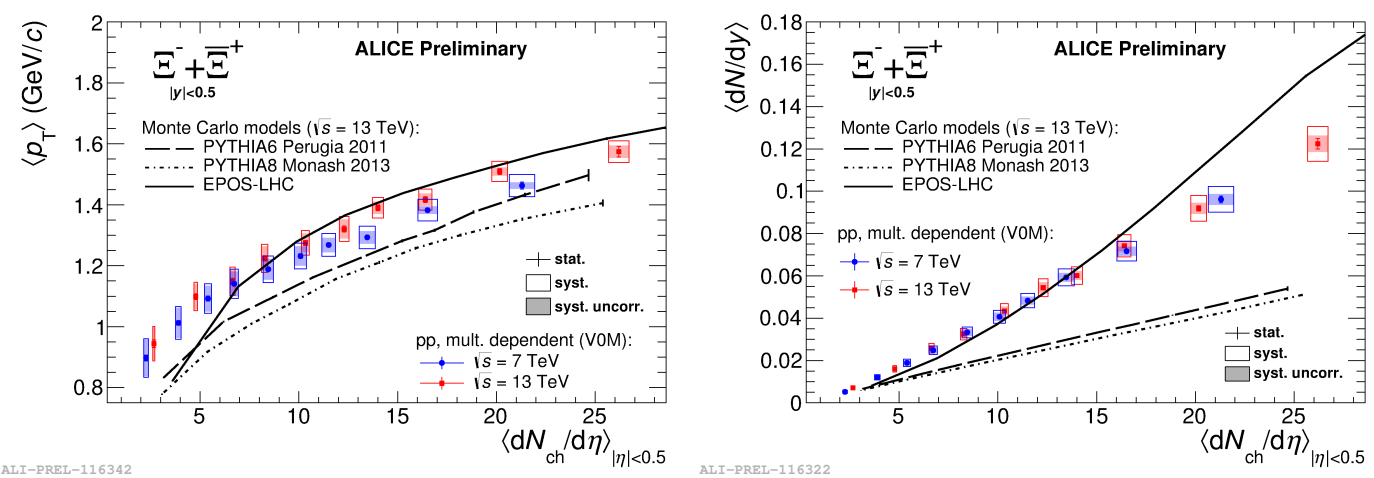

Figure 5: $\left\langle p_{\mathrm{T}}\right\rangle$ (left) and integrated yields (right) of $\Xi$ hyperon as a function of charged particle multiplicity in proton-proton collisions. The shown examples for $\Xi$ are valid also for other particles $\left(\mathrm{K}_{S}^{0}, \Lambda\right.$ and $\Omega$ ). 


\section{Summary}

Intriguing trends are observed for the strangeness production in high multiplicity pp collisions. The results resemble the trends from $\mathrm{Pb}-\mathrm{Pb}$, like e.g. hardening of $p_{\mathrm{T}}$ spectra or differences between baryon-to-meson ratios in lowest and highest multiplicity events. The relative yield of strange particles to charged pions at mid-rapidity is observed to increase with multiplicity in $\mathrm{pp}$ collisions. At a given multiplicity, strangeness production does not depend on collision system and (at least for the LHC) on collision energy; it is purely related to the final state multiplicity. The observed strangeness enhancement is found to follow a hierarchy with strangeness content of the hadron.

\section{References}

[1] J. Rafelski and B. Müller, Phys. Rev. Lett. 48 (1982) 1066-1069

[2] F. Antinori et al. (NA57 Collaboration), J. Phys. G 32 (2006) 427

[3] B. I. Abelev et al. (STAR Collaboration), Phys. Rev. C 77 (2008) 044908

[4] B. Abelev et al. (ALICE Collaboration), Phys. Lett. B 728 (2014) 216

[5] S. Hamieh, K. Redlich and A. Tounsi, Phys. Lett. B 486 (2000) 61-66

[6] CMS Collaboration, JHEP 09 (2010) 091

[7] CMS Collaboration, Phys. Lett. B 718 (2013) 795

[8] B. Abelev et al. (ALICE Collaboration), Phys. Lett. B 719 (2013) 29

[9] J. Adam et al. (ALICE Collaboration), Nature Physics 13 (2017) 535

[10] B. Abelev et al. (ALICE Collaboration), Int. J. Mod. Phys. A 29 (2014) 1430044

[11] R. Fries and B. Müller, Eur. Phys. J. C 34 (2004) S279

[12] K. Aamodt et al. (ALICE Collaboration), Phys. Rev. Lett. 106 (2011) 032301

[13] C. Bierlich and J. R. Christiansen, Phys. Rev. D 92 (2015) 094010

[14] G. Agakishiev et al. (STAR Collaboration), Phys. Rev. Lett. 108 (2012) 072301

[15] J. Adams et al. (STAR Collaboration) Phys. Rev. Lett. 98 (2007) 62301 